in an XY chromosome pair, though in all other cases it requires also a recessive partner. Numerically, on the simplest assumptions, we should expect the variability due to the sex chromosome in the heterogametic sex to be increased by about 9.86 per cent., while that of the homogametic sex should be diminished by 27.47 per cent. The difference is $5 \mathrm{I} \cdot 5$ per cent. of the smaller value, and on comparing this with our observed difference of 38 per cent it will be seen that either additional causes must be sought for the striking excess of female variation, or the greater part of the variation present must be ascribed to the sex chromosome, which is unlikely.

Whatever may be the explanation of this great apparent activity of the sex chromosome, the two main inferences from the statistical study of the effect of Mendelian factors under selection, (I) that variability in any one locality is greater for the more numerous species, (2) that it is greater in the heterogametic sex, have both been unmistakably verified in the body of material which we have examined.

Rothamsted Exp. Station. R. A. FISHER.

Wadham College, Oxford.

E. B. FORD.

\section{The Atomicity of Electricity as a Quantum Theory Law.}

IN the five-dimensional theory of the connexion between electromagnetism and gravitation first proposed by Th. Kaluza (Sitzungsberichte d. Berl. Akad., 192x, S. 766; see also O. Klein, Zs. für Phys, 37, 875, 1926), the equations of motion of an electrified particle may be shown to be the equations of geodetics belonging to the following line element:

$$
d \sigma=\sqrt{\left(d x^{o}+\beta \phi_{i} d x^{i}\right)^{2}+g_{i k} d x^{i} d x^{k}},
$$

where $x^{1}, x^{2}, x^{3}, x^{4}$ are the co-ordinates of ordinary space time with the line element $g_{i k} d x^{i} d x^{k}$, while $x^{\circ}$ is a fifth co-ordinate, and the $\phi_{i}$ are the four co-variant components of the electromagnetic potential vector. If the constant $\beta$ is given the value

$$
\beta=\sqrt{2 \kappa} \text {, }
$$

* being the Einstein gravitational constant, the I4 field equations of the Einstein theory may, moreover, be simply expressed by means of the curvature tensor belonging to this line element.

Let now $d \tau$ be the differential of proper time belonging to a particle of mass $m$ and charge $e$; then the Lagrange function $L$ for the geodetics representing the motion of the particle may be given the form

$$
L=\frac{1}{2} m\left(\frac{d \sigma}{d \tau}\right)^{2}
$$

Defining momenta in the ordinary way by putting

$$
p_{i}=\frac{\partial L}{\partial\left(d x^{i} / d t\right)} \quad(i=0,1,2,3,4)
$$

$p_{0}$ is seen to be constant along a geodetic, since $x^{\circ}$ does not appear in $L$. In addition to the equation expressing this constancy, the system belonging to (3) contains four equations which indeed become identical with the equations of motion of the particle if we put

$$
p_{0}=\frac{e}{\beta c} .
$$

With this choice of $p_{0}$ the momenta $p_{1}, p_{2}, p_{3}, p_{4}$ are further seen to agree with the ordinary definition of the momenta of an electrified particle.

Now the charge $e$, so far as our knowledge goes, is always a whole multiple of the electronic charge, so that we may write

$$
p_{0}=\frac{N \epsilon}{\beta c},
$$

$\epsilon$ being the electronic charge and $N$ a whole number, positive or negative. This formula suggests that the atomicity of electricity may be interpreted as a quantum theory law. In fact, if the five-dimensional space is assumed to be closed in the direction of $x^{\circ}$ with a period $l$, and if we apply the formalism of quantum mechanics to our geodetics, we shall expect $p_{0}$ to be governed by the following rule:

$$
p_{0}=N_{\dddot{l}}^{h}, .
$$

$N$ being now a quantum number, which may be positive or negative according to the sense of motion in the direction of the fifth dimension, and $h$ the constant of Planck. Comparing (7) with (6), and making use of the value (2) of $\beta$, we get for the period $l$ :

$$
l=\frac{h c \sqrt{2 \kappa}}{\epsilon}=0.8 \times \mathrm{IO}^{-30} \mathrm{~cm} .
$$

The small value of this length together with the periodicity in the fifth dimension may perhaps be taken as a support of the theory of Kaluza in the sense that they may explain the non-appearance of the fifth dimension in ordinary experiments as the result of averaging over the fifth dimension.

In a former paper $(Z s$. für Phys., l.c.) the writer has shown that the differential equation underlying the new quantum mechanics of Schrödinger can be derived from a wave equation of a five-dimensional space, in which $h$ does not appear originally, but is introduced in connexion with a periodicity in $x^{\circ}$. Although incomplete, this result, together with the considerations given here, suggests that the origin of Planck's quantum may be sought just in this periodicity in the fifth dimension.

Copenhagen, September 3.

\section{The Rôle of the Cerebellum in the Co-ordination of Animal Movement.}

THE intracerebellar nuclei, forming, as they do, important stations on the course of the cerebellar reflex arcs, naturaily invite inquiry as to the kind of influence they exert on the muscles. Our observations have been made on the cat, decerebrated in deep anæsthesia according to Sherrington's original technique, the plane of transection of the neuraxis passing just in front of the superior colliculus and in front of the infundibulum, thus leaving intact the nucleus ruber. Horizontal slices were then removed from the cerebellum on one or both sides, so as to expose for stimulation the dorsal surfaces of the nuclei. Measures were taken to arrest the bleeding and to maintain the proper temperature. The approximate position of the nucleus in question having been determined by measurements, the section of cerebellum in this neighbourhood was then carefully explored with minimal currents applied by a unipolar electrode. The following is a brief résumé of out results obtained by stimulation of the several nuclei.

Faradisation of the outer side of the nucleus dentatus.-This yielded repeated flexions at the elbow of the ipsilateral foreleg, when the limb was already somewhat flexed owing to the cerebellar removal; the limb was also abducted. Results of this kind were obtained by Horsley and Clarke. The contralateral foreleg, previously in slight extension, showed adduction with palmar flexion of paw. The 